\title{
Listening with a dual brain: Hemispheric asymmetry in sustained attention
}

\author{
JOEL S. WARM, DAVID O. RICHTER, RONALD L. SPRAGUE, \\ PHILLIP K. PORTER, and DONALD A. SCHUMSKY \\ University of Cincinnati, Cincinnati, Ohio 45221
}

\begin{abstract}
Subjects listened for increments in the duration of recurrent white-noise pulses during an 80-min vigil. Critical signals for detection appeared in either a temporally regular or an irregular manner while acoustic stimulation was delivered either binaurally or monaurally to the left or to the right ear. Temporal regularity had no significant effect on performance, but performance was influenced by the input conditions and by time on task. Response times (RTs) to the detection of binaural and left-ear signals had a common origin and increased in a negatively accelerated fashion over time. By contrast, RTs to right-ear signals were relatively sluggish early in the vigil and remained stable over time. The results suggest a functional asymmetry in hemispheric organization during the maintenance of sustained attention.
\end{abstract}

A substantial array of evidence is available to indicate that the two cerebral hemispheres are differentially specialized for processing information (Corballis \& Morgan, 1978). For the most part, it appears that the left hemisphere dominates in the processing of verbal material and does so in an analytic manner, while the right hemisphere may be dominant for several nonverbal functions and handles this information in a holistic manner (Bever, 1975; Moscovitch, 1973). It is noteworthy that these findings come from situations in which subjects worked under relatively short-term, alerted conditions. Little data are available with regard to cerebral dominance in the processing of information in situations requiring prolonged attention. Yet, as Jerison (1977) has pointed out, the capacity to maintain some level of alertness during prclonged activities represents a fundamental element of everyday life.

A recent study by Warm, Schumsky, and Hawley (1976) represents one of the few attempts to explore the information-handling characteristics of the cortical hemispheres in a sustained attention or vigilance task. They attacked the problem experimentally through the use of acoustic stimuli. Subjects were asked to listen continuously for occasional increments in the duration of whitenoise pulses during a $1-\mathrm{h}$ vigil. Since the auditory system is constructed so that each ear connects predominantly to the contralateral hemisphere (Casseday \& Diamond, 1977; Rosenzweig, 1951), asymmetries in hemispheric organization were inferred from differences in the detection of critical signals delivered to the right and left ears.

Warm et al. (1977) found that when they increased the rate of presentation of critical signals from 24 to 96 signals/h, there was a corresponding drop of $124 \mathrm{msec}$ in

Requests for reprints should be sent to Joel S. Warm, Department of Psychology, University of Cincinnati, Cincinnati, Ohio 45221 . the response time (RT) to detect left-ear signals. By contrast, RTs to right-ear signals decreased by less than half this amount $(54 \mathrm{msec})$. Since increments in the density of critical signals reduce the subject's temporal uncertainty about when such signals will appear (Dember \& Warm, 1979), the results of the experiment led to the conclusion that in a sustained attention situation, the right hemisphere may dominate in processing the temporal properties of signals. The present study continues this line of investigation.

In addition to changes in critical signal density, the temporal uncertainty of signals in a vigilance task can also be manipulated through variations in the intervals of time between signals, or the intersignal intervals (ISIs). These intervals can be made highly regular and predictable or irregular and unpredictable. In general, the speed and accuracy of signal detections is greater under conditions of regular compared with irregular signal presentations (Dember \& Warm, 1979). If the functional asymmetry reported by Warm et al. (1976) is general, one might expect that the left ear/right hemisphere would be more sensitive to variations in signal regularity than the right ear/left hemisphere. One purpose of the present investigation was to test this possibility.

Perhaps the most ubiquitous aspect of performance in a task demanding sustained attention is the decrement function, the tendency for quality of performance to deteriorate with time on watch. In their study of hemispheric asymmetry, Warm et al. (1976) also noted the familiar vigilance decrement; RTs to correct detections grew more sluggish as the vigil progressed. However, this effect was unrelated to ear of presentation. A result of this sort has potentially important implications. It suggests that both hemispheres may participate equally in the maintenance of alertness over time. It also has relevance for theories of vigilance, such as the expectancy and arousal positions, which attempt to incorporate the 
effects of the temporal uncertainty of signals and time on task within a common explanatory framework (cf. Warm, 1977). The finding of ear/hemisphere asymmetry with respect to one of these parameters but not the other implies that the effects associated with them may be mediated by different rather than by common processes.

Granted the potential implications of this pattern of results, it should be examined carefully. More specifically, the experiment in which it was obtained measured the vigilance decrement over only two periods of watch, each $30 \mathrm{~min}$ in length. It is conceivable that such a time base was too coarse to provide an adequate assessment of hemispheric organization in the maintenance of susstained attention. Accordingly, a second purpose of the present study was to provide a more fine-grained analysis of the role of hemispheric asymmetries in the vigilance decrement.

\section{METHOD}

Two groups of 36 subjects participated in the experiment. One of these (the monaural group) was designed to assess hemispheric asymmetry through the presentation of stimulation to either the right or the left ear. It consisted of individuals selected for lateral dominance, in order to maximize the possibility of finding hemispheric differences. They were all right-dominant as indexed by a minimum laterality quotient of +.67 on the Edinburgh handedness inventory (Oldfield, 1971) and by consistent "right" preferences in tests of eye and foot dominance. Subjects in the second group (the binaural group) were not specially selected for lateral dominance and received stimulation simultaneously in both ears. This group served in conditions more representative of the usual vigilance experiments and offered a control against which to compare the performance of subjects in the monaural group. All of the subjects who served in the experiment were undergraduate students at the University of Cincinnati. They were free of any known hearing impairments and took part in order to fulfill a course requirement.

All subjects participated in an 80-min session, during which they listened continuously for occasional increments in the duration of recurrent white-noise pulses. Throughout the session, .5 -sec pulses of stimulation were presented once every $2.0 \mathrm{sec}$. These constituted nonsignal events, to which no overt response was required. A $1-\mathrm{sec}$ pulse (twice the normal duration) was the critical signal for detection. Subjects responded to critical signals by depressing a microswitch held in the right hand. Acoustic stimulation was delivered through a pair of headphones at an intensity of $78 \mathrm{dBA}$ SPL. Appropriate switching circuitry permitted the delivery of white-noise pulses either binaurally or to the left and right ears separately. The auditory task used in this study was identical to that employed by Warm et al. (1976).

The vigil was divided into four 20-min periods of watch. Twenty critical signals were presented in each period in either a regular or an irregular manner. Half of the subjects in both the binaural and monaural groups were assigned at random to one of the two conditions of critical signal regularity. In the regular condition, critical signals appeared once every $60 \mathrm{sec}$; in the irregular condition, they appeared at intervals of $10,35,60,85$ and $110 \mathrm{sec}(\mathrm{mean}=60 \mathrm{sec})$. For each subject who experienced the irregular signal schedule, these ISIs were block randomized every $5 \mathrm{~min}$ throughout the four 20 -min periods of watch. Block randomizations differed within periods for each subject; they also varied nonsystematically across subjects.

For subjects in the monaural group, acoustic pulses were presented to each ear alone for alternating 5 -min periods within each 20 -min period of watch. Equal numbers of critical signals appeared in the left and right ears within each 20 -min period. The sequence in which subjects experienced left-ear/right-ear alternations was balanced within the two conditions of signal regularity.

The occurrence of neutral pulses and critical signals was controlled by solid state programming equipment and a punched-tape timer. Response times to correct detections were read to the nearest $1 \mathrm{msec}$ from a digital electronic timer that was activated automatically $.5 \mathrm{sec}$ after the onset of a pulse comprising a critical event and terminated by the subject's response or after a no-response duration of $2.5 \mathrm{sec}$. Failure to respond to a critical signal within $2.5 \mathrm{sec}$ after its termination was considered an error of omission (missed signal), and responses made in the absence of a critical signal were considered errors of commission or false alarms. The latter were tallied automatically by an electronic counter. Each subject was tested individually in a sound-shielded room; the control equipment was located in an adjacent room. Subjects surrendered their watches upon entering the laboratory; they had no knowledge of the length of the vigil other than that it would not exceed $2 \mathrm{~h}$.

\section{RESULTS}

\section{Binaural Group}

Median RTs to correct detections were computed from the data of each subject receiving binaural stimulation. The data are presented in the left panel of Figure 1. Means of median RTs for the two conditions of signal regularity are plotted as a function of periods of watch.

An analysis of variance of the binaural data revealed that response latencies increased significantly as time on watch progressed and, thus, that performance efficiency suffered a significant deterioration over time $[F(3,102)=$ $3.36, \mathrm{p}<.025]$. Inspection of Figure 1 suggests that RTs in the binaural group were more rapid for regular compared with irregular signals. However, this difference lacked statistical significance $[F(1,34)=1.66, p>.05]$, as did the interaction between regularity and periods $(\mathrm{F}<1)$.

\section{Monaural Group}

Median RTs to correct detections were computed from the data of each subject in the monaural group.

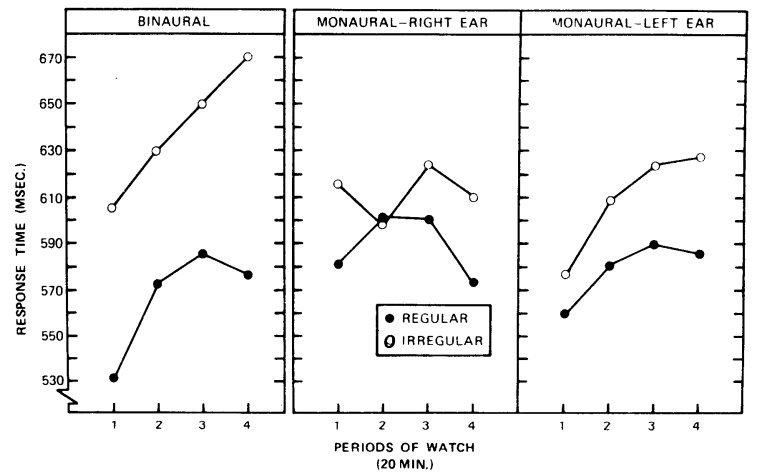

Figure 1. Response times to correct detections for regular and irregular signals as a function of periods of watch. Data for binaural presentations are displayed in the left panel; data for right-ear and left-ear presentations appear in the middle and right panels, respectively. 
Means of median RTs for right and left ear signals are displayed in the middle and right panels of Figure 1, respectively. In both panels, RTs for the two conditions of signal regularity are presented as a function of periods of watch. An analysis of variance of the monaural data revealed that ear of input did not have a significant overall effect on the speed of signal detections $(F<1)$. While Figure 1 shows a tendency for RTs to signals in either ear to be faster for regular compared with irregular signals and for RTs in each ear to follow a different pattern over time, the analysis of variance revealed that only the Ear by Periods interaction reached statistical significance $[F(3,102)=7.02, p<.001]$. All other sources of variance in the analysis lacked significance ( $p>05$ in each case).

The significant interaction between ear of input and periods of watch is one of primary significance for this investigation. It indicates an ear/hemisphere asymmetry in the nature of the vigilance decrement. This interaction is presented in Figure 2. For purposes of comparison, the overall course of performance over time for subjects receiving binaural stimulation is also presented in this figure.

Figure 2 reveals a striking similarity in the time course of performance when subjects listened for critical signals binaurally or with only the left ear. In these conditions,

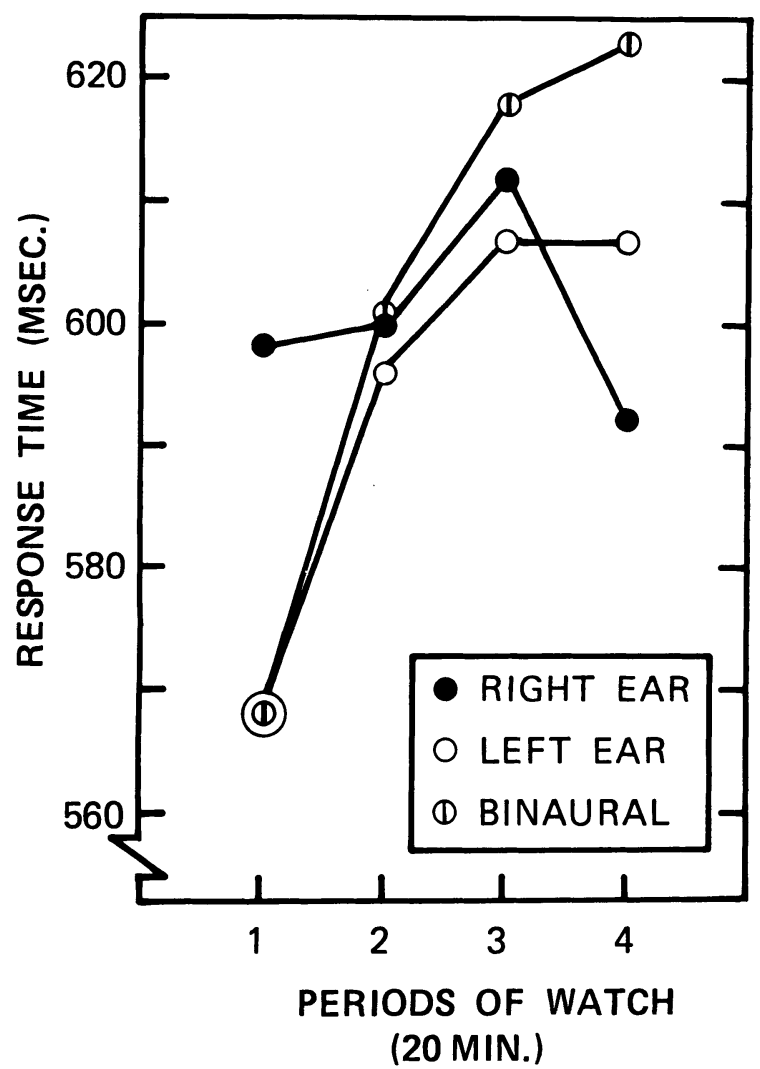

Figure 2. Response times to correct signal detections for binaural, right-ear, and left-ear presentations as a function of periods of watch.
RTs had a common origin during the initial period of watch and increased in a similar, negatively accelerated manner over time. By contrast, when subjects listened for critical signals with the right ear, RTs were relatively sluggish during the early portion of the vigil and remained stable as time on watch progressed. ${ }^{1}$

\section{DISCUSSION}

Although RTs in both the binaural and monaural groups tended to be faster for regular compared with irregular signal presentations, none of the effects involving signal regularity were statistically significant. Consequently, the question of whether one cerebral hemisphere is more sensitive to variations in signal regularity than the other must remain moot for now.

It should be noted that while several experiments have found that the efficiency of vigilance performance is related to variations in signal regularity (Dember \& Warm, 1979), such a relation is not always evident (Boulter \& Adams, 1963). In this regard, we should point out that the irregular ISIs used in this investigation were identical to those employed by McCormack \& Prysiaznuik (1961) in a study that did report significant differences in RTs as a function of signal regularity. However, that experiment required subjects to detect the onset of a brief flash of light, whereas in the present case subjects responded to a change in the duration of acoustic pulses. Evidently, the effects of variations in signal regularity are complex; efficient utilization of cues to the temporal structure of signal occurrences may depend not only on the variability of the ISIs involved, but also upon the sensory modality of signals and the perceptual tasks entailed in responding to them.

Consistent with the general finding in vigilance studies, performance efficiency in both the binaural and monaural groups became progressively degraded over time. The important aspect of this change in efficiency was the fact that an ear/hemisphere asymmetry was evident in the manner in which it occurred. The increment in RTs for leftear/right-hemisphere signals closely mirrored that noted in the binaural control condition, whereas RTs to right-ear/left-hemisphere signals remained essentially stable over time. These results confirm the suspicion that the time base used in the study by Warm et al. (1976) may have been too coarse to provide an adequate assessment of hemispheric differences in the decrement function. They also point to the possibility that the right hemisphere is predominantly involved in controlling the course of sustained attention. However, the fact that the task could be performed with signals delivered to the right ear/left hemisphere in a remarkably stable manner should not be overlooked. Perhaps the most reasonable conclusion to be drawn from the present data is that the two hemispheres may be jointly involved in organizing behavior in a vigilance task and that the final outcome is some weighted average of the activities of each. While the right hemisphere predominates, the more stable action of the left hemisphere might supplement that of the right as the efficiency of the latter degrades with time.

An account of the present results along these lines fits nicely with a proposal made by Levy (1977) concerning the adaptive significance of cerebral asymmetry in information processing. Levy takes the position that cerebral activity is fatiguing to a hemisphere and that performance falls as fatigue increases. If both hemispheres were symmetrically competent with regard to a task at hand, there would be no reserve capacity to counter increments in fatigue. With asymmetrical competence, one side can take over for the other, so that fatigue-induced deterioration is retarded.

In considering the outcome of this investigation, it is essential to note that it and the study by Warm et al. (1976) do not represent the only experimental efforts to assess cerebral dominance in sustained attention. Recently, Diamond (1979a, 1979b) has studied vigilance performance in split-brain patients in whom 
neural connections between the hemispheres had been severed. These individuals consistently performed more efficiently with right- compared with left-hemisphere signals in tasks requiring the detection of auditory, tactual, and visual stimuli.

Taken together, Diamond's $(1979 a, 1979 b)$ results and those of our experiments provide impressive converging evidence that sustained attention may be more a right-brain than a leftbrain function. Nevertheless, a word of caution is in order. Nonverbal stimulation was used in Diamond's work and in ours. Since the right brain dominates the processing of these kinds of stimuli under alerted conditions, the conclusion that vigilance is controlled predominantly by the right brain may be premature. In order to draw a more firm conclusion, it will be necessary to examine sustained attention in more verbally oriented tasks. Such an approach offers interesting possibilities for future research.

\section{REFERENCES}

Bever, T. G. Cerebral asymmetries in humans are due to the differentiation of two incompatible processes: Holistic and analytic. Annals of the New York Academy of Sciences, 1975, 263, 251-262.

Boulter, L. R., \& Adams, J. A. Vigilance decrement, the expectancy hypothesis and intersignal interval. Canadian Journal of Psychology, 1963, 17, 201-210.

Casseday, J. H., \& Diamond, I. T. Symmetrical lateralization of function in the auditory system of the cat: Effects of unilateral ablation of cortex. Annals of the New York Academy of Sciences, 1977, 299, 255-263.

Corballis, M. C., \& Morgan, M. J. On the biological basis of human laterality: I. Evidence for a maturational left-right gradient. The Behavioral and Brain Sciences, 1978, 2, 261-269.

Dember, W. N., \& WARM, J. S. Psychology of perception (2nd ed.). New York: Holt, Rinehart, \& Winston, 1979.

Diamond, S. J. Performance by split-brain humans on lateralized vigilance tasks. Cortex, 1979, 15, 43-50. (a)

DiAmond, S. J. Tactual and auditory vigilance in split-brain man. Journal of Neurology, Neurosurgery and Psychiatry, 1979, 42, 70-74. (b)

JERISON, H. J. Vigilance: Biology, psychology, theory and practice (keynote address). In R. R. Mackie (Ed.), Vigilance: Theory, operational performance, and physiological correlates. New York: Plenum, 1977.

LEVY, J. The mammalian brain and the adaptive advantage of cerebral asymmetry. Annals of the New York Academy of Sciences, 1977, 299, 264-272.

McCormack, P. D., \& Prysiaznuik, A. W. Reaction-time and regularity of interstimulus interval. Perceptual and Motor Skills, 1961, 13, 15-18.

Moscovitch, M. Language and the cerebral hemispheres: Reactiontime studies and their implications for models of cerebral dominance. In P. Pliner, L. Krames, \& T. Alloway (Eds.), Communication and affect: Language and thought. New York: Academic Press, 1973.

OLDFIELD, R. The assessment and analysis of handedness: The Edinburgh inventory. Neuropsychologia, 1971, 9, 97-113.

Pachella, R. G. The interpretation of reaction time in informationprocessing research. In B. H. Kantowitz (Ed.), Human information processing: Tutorials in performance and cognition. Hillsdale, N.J: Erlbaum, 1974.

Rosenzweig, M. R. Representation of the two ears at the auditory cortex. American Journal of Physiology, 1951, 167, 147-158.

WARM, J. S. Psychological processes in sustained attention. In R. R. Mackie (Ed.), Vigilance: Theory, operational performance, and physiological correlates. New York: Plenum, 1977. Warm, J. S., Schumsky, D. A., \& Hawley, D. K. Ear asymmetry and the temporal uncertainty of signals in sustained attention. Bulletin of the Psychonomic Society, 1976, 7, 413-416.

\section{NOTE}

1. Prior research with this vigilance task using acoustic stimulation has resulted in a very high level of signal detections (cf. Warm et al., 1976). The present experiment was no exception; mean detection probabilities under all conditions ranged from .88 to .99 . No significant relations were noted with the variations of any of the independent variables in regard to the rate of signal detections, and there was' no evidence of any speed-accuracy tradeoffs (cf. Pachella, 1974).

(Received for publication January 26, 1980.) 